\title{
Die Bildung \\ fester Bromoxyde bei der thermischen Reaktion zwischen Brom und Ozon
}

\author{
Bemerkungen zu der voranstchenden Arbeit \\ von A. J. Arvía, P. J. Aymonino und H. J. Schumacher ${ }^{1}$ ) \\ Von A. Pflugmacher
}

Bei der thermischen Reaktion zwischen Brom und Ozon erhielten wir reproduzierbar und eindeutig ein weißes Bromoxyd der Zusammensetzung $\mathrm{BrO}_{3}$. Wir bestätigten damit unsere früher gemachten Feststellungen, wonach auch in der Glimmentladung aus Brom und Sauerstoff ein Oxyd dieser Zusammensetzung entsteht. Die argentometrisch und jodometrisch gewonnenen Analysenwerte für das Sauerstoff/BromVerhältnis liegen zwischen den Grenzen 2,93 und 3,00 und lassen keinen Zweifel an der Einheitlichkeit der Verbindung aufkommen.

Da das von uns dargestellte Oxyd alle Eigenschaften des früher von LewIS und SCHUMACHER ${ }^{2}$ ) beschriebenen $\mathrm{Br}_{3} \mathrm{O}_{8}$ zeigt, glaubten wir die Annahme aussprechen zu dürfen, daß das von LEwIS und SCHUMACHER dargestellte Oxyd mit dem unsrigen identisch sei, obwohl die dort mitgeteilten Analysenwerte von der für das $\mathrm{BrO}_{3}$ geforderten $\mathrm{Zu}$ sammensetzung abweichen.

Vorstehende Veröffentlichung zeigt nun, daß je nach Gefäßmaterial weiße Bromoxyde verschiedener Zusammensetzung zwischen den Grenzwerten $\mathrm{BrO}_{2,45}$ und $\mathrm{BrO}_{2,86}$ entstehen können. Inwieweit es sich dabei um einheitliche Verbindungen oder Gemische handelt, ist aus den Analysendaten nicht eindeutig zu erkennen.

Die Tatsache, daß Sonumacher und Mitarbeiter durch die chemische Analyse stets niedrigere Sauerstoffwerte erhalten als durch die Gasdruckmessung, ist leicht zu erklären. Das Oxyd hat sich dann schon vor der Umsetzung mit der angesäuerten KJ-Lösung teilweise zersetzt, wobei das freigewordene Brom noch aus der Gasphase zur Reaktion gebracht und analytisch erfaßt werden kann, nicht aber der inzwischen

1) A. J. Arvía, F. J. Aymonino u. H. J. Schumach fr, Z. anorg. allg. Chem. 29S, 1 (1958).

2) B. Lewis u, H. J. Schumacirer, Z. anorg. allg. Chem, 182, 182 (1929). 
im molekularen Zustand freigewordene Sauerstoff. Richtige Werte, die den tatsächlichen Verhältnissen entsprechen, erhält man auf diesem Wege nur dann, wenn man die KJ-Lösung durch einen genügend weiten Rohrquersehnitt in das vorher mit flüssiger Luft gekühlte Reaktionsgefäß einströmen läßt, so daß die gesamte Innenwandung sofort mit der KJ-Lösung benetzt wird, wobei letztere gefriert. Das Gelingen des Experiments erkennt man daran, daß nach dem Auftauen des Gefäßinhaltes der über der Flüssigkeit befindliche Gasraum farblos, also frei von Bromdämpfen ist.

Aachen, Institut für Anorganische Chemie und Elektrochemie der Technischen Hochschule.

Bei der Rerlaktion eingegangen am 3. April 1958. 